\title{
Method of the calculated estimation of the possibility of progressive destruction of buildings in result of fire
}

\author{
Serhii Pozdieiev ${ }^{1, *}$, Olha Nekora $^{2}$, Tetiana Kryshtal $^{2}$, Vitalii Zazhoma ${ }^{2}$, and Stanislav \\ Sidnei ${ }^{2}$ \\ ${ }^{1}$ Cherkasy State Technological University (CSTU), 18006, Cherkasy, Shevchenko blvd. 460, Ukraine \\ ${ }^{2}$ Cherkasy Institute of Fire Safety named after Chornobyl Heroes of National University of Civil \\ Defense of Ukraine, 18034 Cherkassy, Onoprienko Str., 8, Ukraine
}

\begin{abstract}
When analyzing the fire safety of building structures, in addition to considering their fire resistance, the limits of flame expansion and other fire-safety engineering features, there should be considered also the probability of their progressive collapse in case of a damage caused to some their elements. In case of progressive collapse of the building structures, socio-economic losses become the worst possible. The article proposes a method of calculation for the estimation of the possibility of progressive destruction. The method is based on the assumption that one or several compressed elements are damaged and must be removed from the system, which ensures the rigidity and geometric immutability. The basic point of the method is the hypothesis of formation of the line of plastic hinge joints in the ceiling plate. The estimation of the possibility of progressive destruction is made according to the energy criterion on the basis of a comparison of the work of internal and external forces on the possible movements of the system, which, under these conditions, is geometrically variable. The proposed method is productive and economical comparing to the existing methods which involve complex mathematical models and software complexes.
\end{abstract}

\section{Introduction}

The statistics of fires and fire-related accidents reveals that one of the most dangerous factors is the destruction of load-carrying reinforced concrete structures, as shown by the works [1-6]. In this case, the destruction reaches its highest level as the damage caused to one or more elements of structures leads to a series of collapses of other elements, resulting in collapse of a part or entire building. In case of progressive destruction, socio-economic losses are the highest possible. One of the effective measures to ensure the survivability of buildings and their structures is the introduction of modern calculation methods for predicting the possibility of progressive destruction and the use of appropriate engineering measures to strengthen the building structures.

\footnotetext{
* Corresponding author: svp chipbbk@ukr.net
} 


\subsection{Literature review}

According to the researches [7], one of the most efficient and economical methods of engineering estimation of the possibility of progressive destruction of buildings and structures is the kinematic method. The method, used in the researches, determines the work of internal forces $(W)$ and external loading $(U)$ for each of the previously adopted mechanisms of progressive destruction on the possible movements of the considered mechanism, into which the static system is transformed. The condition for preserving the static system unchanged is a solution to the inequality:

$$
W \geq U \text {. }
$$

The model of a building frame, which is in critical condition, assumes a complete removal of the destroyed column from the structure of the rigidity of the building. The removed column is not considered anymore to be a part of the mechanism, into which the building is transformed, along with the existing plastic hinge joints in it. At the same time, this method has a certain shortcoming: the lines of plastic hinge joints are straight, while the separated zones between the lines of plastic hinge joints have a form of triangular facets. This approach may lead then to the fact that unreasonable overcharging of the model of plastic hinge joints lines with additional lines may lead to significant distortion of the obtained results and imposes the need to introduce additional coefficients of safety margins for the investigated structures.

Another approach to the calculated forecasting of the progressive destruction is described and efficiently applied in the researches [8]. This approach is based on the application of the finite element method in the combination with the method of penalty functions in separating and co-inflicting of the parts with finite elements, formed again in the process of calculating the emergency system. This approach requires the use of laborintensive and long-term calculations involving complex software and skilled engineers.

\subsection{Purpose, objectives and results}

The purpose of the research (its main results are provided by the article) is developing a mathematical description of the work of external and internal forces in the kinematic model of the system, into which the structure of the building is transformed in case of inserting the plastic hinge joint lines in the ceilings, limiting the curvilinear portions of this geometrically variable system as the basis of the improved kinematic calculation method for predicting the progressive destruction in buildings with reinforced concrete structures.

\section{Basic theoretical provisions for the calculation}

To considerate the progressive destruction, based on the assumptions, there was used a universal calculation model (shown in Fig. 1). This model corresponds to the kinematic calculation of a part of a building with one element, which is assumed to be destroyed in case of fire. It is also taken into account that the destruction of the column occurs after the destruction of the ceiling that may happen in result of a possible fire. 


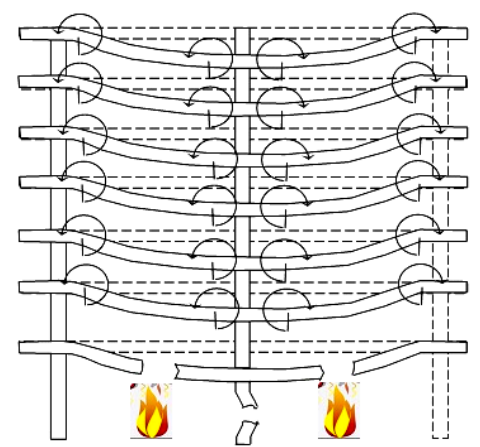

Fig. 1. The calculation model used for calculating the progressive destruction of a building with reinforced concrete structures.

Fig. 2 shows a model of reproduction of the plastic hinge joint lines, according to the Bezier curves. The hypothesis that plastic hinge joint lines can be recreated using the Bezier curves was adopted by the assumption on the location of plastic hinge joints on the tangents, which divide the parallel lines to series of undamaged columns and walls in the percentage correlated with the variable function of the Bezier curves.

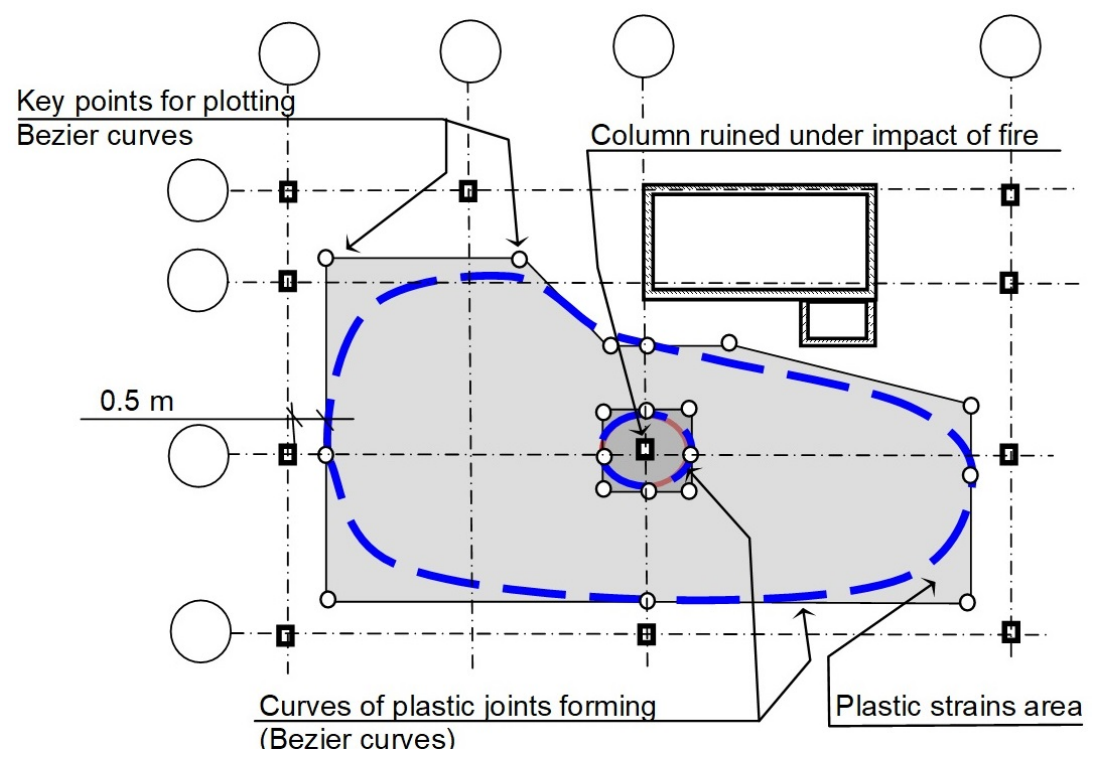

Fig. 2. Reproduction of lines of formation the plastic hinge joint using Bezier curves.

According to Fig. 2, two lines of the formation of plastic hinge joints should be considered: a line around the column (rigidity diaphragm) and a line that envelops the compressed load-bearing elements of the selected part of the building which is subjected to progressive destruction. The application of the approximation is due to the fact that the Bezier curves are continuous to the derivatives of the first and second order, therefore, they allow taking into account the specific features of the zone of plastic deformation and define the current values of the corresponding geometric parameters used for the determination of both internal and external force factors.

The equation of the function describing the Bezier curve is written in an implicit form and represents a system of two parametric equations [9]: 


$$
x(u)=\sum_{k=1}^{n} x_{k} B_{k, n}(u), y(u)=\sum_{k=1}^{n} y_{k} B_{k, n}(u),
$$

where $x_{k}$ and $y_{k}$ are the coordinates of the nodal points for construction of the Bezier curve. In formula (2) the Bernstein polynomials are determined by the formula [9]:

$$
B_{k, n}(u)=\frac{n !}{k !(n-k) !} u^{k}(1-u)^{n-k}
$$

where $n$ - number of control points; $k$ - current number of the nodal point.

\section{Mathematical apparatus to calculate the work of internal forces}

Using expressions (2) and (3), the virtual work of internal forces can be determined according to the models shown in Fig. 3 and Fig. 4.

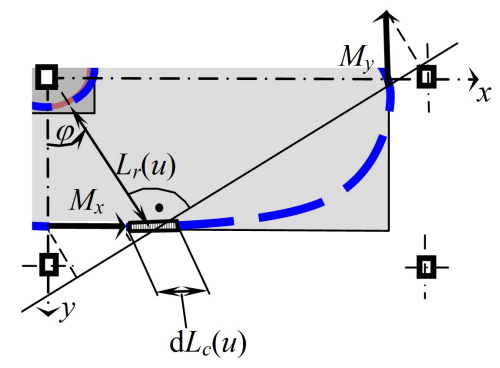

Fig. 3 Model for determining the work of the boundary internal forces in plastic hinge joints of ceiling.

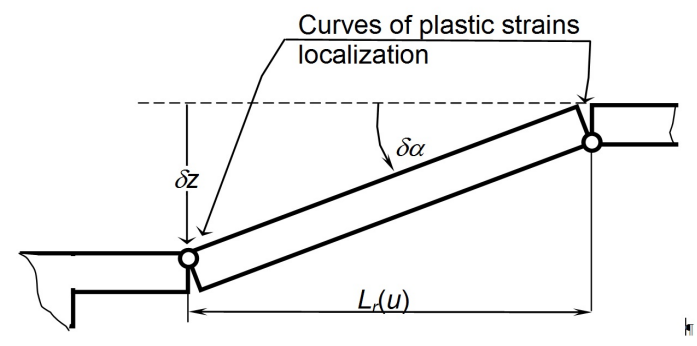

Fig. 4 Model for determining possible displacements in case of determining the work of internal forces in ceilings.

The following calculation models determine the work of the internal forces on the possible displacement $\delta z$, using the following expressions:

$$
\begin{gathered}
W_{s l 1}=\int_{0}^{1}\left(M_{x 1} \cos \varphi+M_{y 1} \sin \varphi\right) L_{c 1}(u) \frac{\delta z}{L_{r}(u)} d u, W_{s l 2}=\int_{0}^{1}\left(M_{x 2} \cos \varphi+M_{y 2} \sin \varphi\right) L_{c 2}(u) \frac{\delta z}{L_{r}(u)} d u, \\
\cos \varphi=\frac{\operatorname{tg} \varphi}{\sqrt{1+\operatorname{tg}^{2} \varphi}}, \sin \varphi=\frac{1}{\sqrt{1+\operatorname{tg}^{2} \varphi}}, \operatorname{tg} \varphi=y(u) / x(u),
\end{gathered}
$$




$$
\begin{gathered}
L_{c 1}(u)=\sqrt{\left(\frac{d x_{1}(u)}{d u}\right)^{2}+\left(\frac{d y_{1}(u)}{d u}\right)^{2}}, L_{c 2}(u)=\sqrt{\left(\frac{d x_{2}(u)}{d u}\right)^{2}+\left(\frac{d y_{2}(u)}{d u}\right)^{2}} \\
L_{r}(u)=\sqrt{\left(x_{1}(u)\right)^{2}+\left(y_{1}(u)\right)^{2}}-\sqrt{\left(x_{2}(u)\right)^{2}+\left(y_{2}(u)\right)^{2}}, W=W_{s 11}+W_{s l 2},
\end{gathered}
$$

where $M_{x}, M_{y}$ are the lateral boundary moments during the plastic boundary deformation of ceiling slabs, determined by the method of boundary deformations.

The first derivatives of the functions that describe Bezier curves are determined by the following expressions [6]:

$$
\frac{d x(u)}{d u}=\sum_{k=1}^{n} x_{k} B_{k, n}^{\prime}(u), \frac{d y(u)}{d u}=\sum_{k=1}^{n} y_{k} B_{k, n}^{\prime}(u), B_{k, n}^{\prime}(u)=B_{k, n}(u) \frac{k-n u}{u(1-u)}
$$

When implementing this calculation method, a value of the variable should be obtained to determine the function of the Bezier curve around the vertical supporting element, corresponding to the current angle $\varphi$. For this purpose, the following formulas can be applied:

$$
\begin{gathered}
x_{2}(u)=\sum_{k=1}^{n} x_{k} B_{k, n}(p(u)), y_{2}(u)=\sum_{k=1}^{n} y_{k} B_{k, n}(p(u)), \\
p(u)=\frac{p_{1}(u)-p_{2}(u)+\sqrt{p_{2}^{2}-p_{1}(u) p_{3}(u)}}{p_{1}(u)-2 p_{2}(u)+p_{3}(u)}, p_{k}(u)=y_{k}-x_{k} \frac{y_{1}(u)}{x_{1}(u)} .
\end{gathered}
$$

\section{Mathematical apparatus to calculating external forces work}

The following calculation models determine the work of internal forces in one quadrant on the possible displacement $\delta z$ using the following expressions:

$$
\begin{gathered}
U_{s l}=U_{s l 1}+U_{s l 2}, U_{s l 1}=\frac{1}{6} \eta_{f i} Q_{s l} \delta z \int_{0}^{1} L_{c 1}(u) L_{r 1}(u) d u-\frac{1}{2} \eta_{f i} Q_{s l} \delta \int_{0}^{1} \frac{L_{c 2}(u) L_{r 2}(u)}{L_{r}(u)}\left(\frac{1}{3} L_{r 2}(u)+L_{r}(u)\right) d u \\
U_{s l 2}=\frac{1}{2} \eta_{f i} Q_{s l} \delta z \int_{0}^{1} L_{c 2}(u) L_{r 2}(u) d u-0.25 \eta_{f i} Q_{s l} \delta z \cdot S_{c} \\
L_{r 1}(u)=\sqrt{\left(x_{1}(u)\right)^{2}+\left(y_{1}(u)\right)^{2}}, L_{r 2}(u)=\sqrt{\left(x_{2}(u)\right)^{2}+\left(y_{2}(u)\right)^{2}}
\end{gathered}
$$

We also need to determine the work of external forces due to the own weight of the columns. For this purpose the following formula is applied:

$$
U_{c}=\left(\rho_{c} A_{c}+\rho_{s} A_{s}\right) L_{c}(1+1 / N) \delta z
$$

where $\rho_{c}$ - density of concrete; $\rho_{s}$ - density of reinforcement steel; $N$ - number of ceilings that were not destroyed by fire; $S_{c}$ - cross-sectional area of a column or a diaphragm of stiffness; $S_{s}$ - cross-sectional area of the column reinforcement or a diaphragm of stiffness; $L_{p}$ - length of the column within the framework of the floor.

The general work of external forces in possible displacements is as follows:

$$
U=U_{s l}+U_{c}
$$


Integration of the expressions (4), (11) and (12) is performed by one of the numerical methods. In this particular case, it is recommended to apply the Romberg's method [10].

\section{Calculation method of estimation the progressive destruction possibility}

For the calculation aimed at determining the possibility of progressive destruction of a building in result of fire, the following procedures should be performed.

1. One or a group of columns (diaphragms) is determined to be removed from the structure of the rigidity of the building as destroyed in result of fire. 2 . The points of the boundary of the zone of plastic deformations for the first and second lines of plastic hinge joints are to be determined. The obtained zone should have boundaries passing through the points located $0.5 \mathrm{~m}$ away from groups of undamaged columns and rigidity diaphragms. The obtained zone is divided into several parts by the axial lines drawn through the center of gravity of the column section or the sections of a group of columns. 3 . The boundary moments in the ceiling slabs are determined on conditions of normal temperatures. 4 . The location of the points, through which the curves of local plastic deformation should pass (the Bezier line), are to be determined. The coordinate vector of these points is written for the parametric functions, which are described in accordance with the formula (2). 5. Applying the formulas (4)-(10), there is determined the possible work of the internal forces in each of the parts, into which the zone of plastic deformations, located around the removed columns, was previously divided. The total amount of possible work is defined as the sum of all obtained components. 6. Applying the formulas (11) and (15), the summed amount of the possible work of external forces is defined. 7. A performance of the condition (1) is checked and a conclusion on the possibility of progressive destruction of the building in case of fire is drawn.

\section{Conclusions}

As a result of the research, the following results were obtained:

- there was developed a mathematical apparatus for the determination of virtual work for the external and internal forces of kinematic systems, into which the structural systems of buildings, including the ones in disrepair, are transformed as a result of a fire;

- on the basis of the proposed mathematical apparatus there was improved an advanced kinematic calculation method for predicting the possibility of progressive destruction.

\section{References}

1. S. Pozdieiev, O. Nuianzin, S. Sidnei, S. Shchipets Computational study of bearing walls fire resistance tests efficiency using different combustion furnaces configurations MATEC Web of Conferences, 116, 02027, (2017).

2. O. Nekora, V. Slovynsky, S. Pozdieiev The research of bearing capacity of reinforced concrete beam with use combined experimental-computational method MATEC Web of Conferences, 116, 02024, (2017).

3. Y. Danchenko, V. Andronov, M. Teslenko, V. Permiakov, E. Rybka, R. Meleshchenko, A. Kosse Study of the free surface energy of epoxy composites using an automated measurement system EasternEuropean Journal of Enterprise Technologies, 1, (12-91), (2018). 
4. B. Pospelov, V. Andronov, E. Rybka, V. Popov, A. Romin Experimental study of the fluctuations of gas medium parameters as early signs of fire. EasternEuropean Journal of Enterprise Technologies, 1, (10-91), (2018).

5. B. Pospelov, V. Andronov, E. Rybka, Vadym Popov, O. Semkiv Development of the method of frequency-temporal representation of fluctuations of gaseous medium parameters at fire 2, 10, (2018).

6. DBN V.1.1-7-2016: State Building of Ukraine, Fire protection. Building structures fire safety (2017).

7. G.I. Shapiro, Yu.A. Eisman, A.S. Zalesov, Rekomendacii po zachite monolitnyh zdanii ot progressiruyuchego obrushenia. Moskomarchitectury. Moscow. 28, (2005).

8. J.O. Hallquist: LS-DYNA Theory Manual, Livermore Software Technology Corporation: California, USA, 680, (2005).

9. D Hearn., M.P. Baker, W.R. Carithers Computer graphics with Open GL. 4-th edition. Harlow: Pearson Education Limited, 812, (2014).

10. N. Piskunov Differential and integral calculus for technical institutes. 13-th edition Moscow: Science, Main redaction of technical literature. 560, (1985) 